Article

\title{
Five New Limonoids from Peels of Satsuma Orange (Citrus reticulata)
}

\author{
Takashi Kikuchi, Yasuaki Ueno, Yoshino Hamada, Chika Furukawa, Takako Fujimoto, \\ Takeshi Yamada and Reiko Tanaka * \\ Faculty of Pharmaceutical Sciences, Osaka University of Pharmaceutical Sciences, 4-20-1 Nasahara, \\ Takatsuki, Osaka 569-1142, Japan; t.kikuchi@gly.oups.ac.jp (T.K.); e10331@gap.oups.ac.jp (Y.U.); \\ yoshinotama@yahoo.co.jp (Y.H.); chika1026@pearl.ocn.ne.jp (C.F.); e11537@gap.oups.ac.jp (T.F.); \\ yamada@gly.oups.ac.jp (T.Y.) \\ * Correspondence: tanakar@gly.oups.ac.jp; Tel.: +81-72-690-1084
}

Academic Editor: Derek J. McPhee

Received: 21 April 2017; Accepted: 26 May 2017; Published: 31 May 2017

\begin{abstract}
Five new: 21,23-dihydro-21-hydroxy-23-oxonomilin (1), 21,23-dihydro-23-methoxy-21oxonomilin (2), 21,23-dihydro-21-hydroxy-23-oxonomilinic acid methyl ester (3), 21,23-dihydro-23methoxy-21-oxolimonin (4), and 21,23-dihydro-21-oxolimonin (5), and seven known limonoids were isolated from peels of satsuma orange (Citrus reticulata). The isolated compounds were evaluated for their inhibitory effects on macrophage activation by an inhibitory assay of nitric oxide (NO) production. Among them, compound (2) exhibited NO inhibitory activity without cytotoxicity.
\end{abstract}

Keywords: limonoid; Rutaceae; satsuma orange; Citrus reticulata; inhibitory activity on nitric oxide production

\section{Introduction}

Satsuma orange (Scientific name: Citrus reticulata Blanco; Synonym: Citrus unshiu Marcov.; Japanese name: unshu mikan), belongs to Rutaceae, and is cultivated in Japan. The peels of its fruits, called chinpi, have been used as an aromatic stomachic, cold medicine, expectorant, and antitussive [1]. Limonoids [2,3], flavonoids [4-7], tocopherol analogues [8,9] have been isolated from peels of C. reticulata, phenyl glycosides from flower buds [10], and a limonoid [11], flavonoids [11], and a cyclic peptide from its fruits [12]. Moreover, some bioactivities of constituents from C. reticulata have been reported, such as the suppression of adipogenesis by a limonoid [3], the inhibitory activity on histamine release from rat peritoneal mast cells [4], hypotensive activity [5] and antioxidant activity [6] of flavonoids, the radical-scavenging activity [8] and hepatoprotective and neuroprotective activities [9] of tocopherol analogues, as well as antimicrobial activities of essential oil [13]. In the present study, we isolated five new limonoids 1-5; 21,23-dihydro-21-hydroxy-23-oxonomilin (1), 21,23-dihydro-23-methoxy-21-oxonomilin (2), 21,23-dihydro-21-hydroxy-23-oxonomilinic acid methyl ester (3), 21,23-dihydro-23-methoxy-21-oxolimonin (4), and 21,23-dihydro-21-oxolimonin (5), along with known compounds (6-12). Compounds 1-12 were evaluated for inhibitory effects of limonoids on nitric oxide $(\mathrm{NO})$ production by macrophages.

\section{Results and Discussion}

Five new (1-5), and seven known compounds (6-12) were isolated from the $C$. reticulata peels (Figure 1). The known compounds were identified as limonin (6) [14,15], shihulimonin A (7) [16], limonexic acid (8) [17], evolimorutanin (9) [18], kihadanin A (10) [19], deacetylnomilin (11) [20], and ichangin (12) [21] by comparison of their spectroscopic data with those previously reported. 


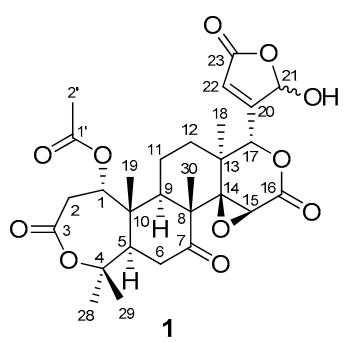

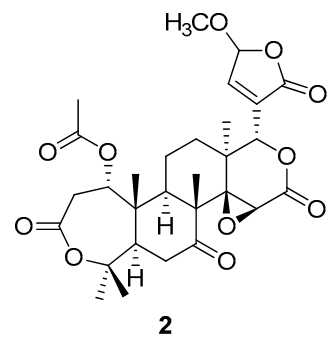

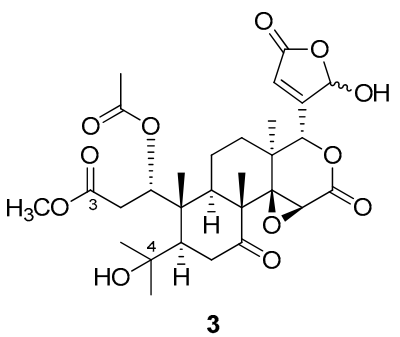

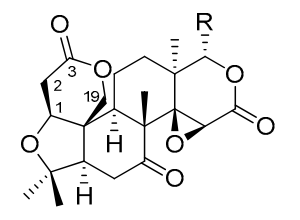<smiles>CCC1=CC(OC)OC1=O</smiles><smiles>[BH3-]</smiles><smiles>CCC1=CCOC1=O</smiles><smiles>[20BH2]</smiles><smiles></smiles><smiles>CC(C)C1=C[C@@H](O)OC1=O</smiles>

C:<smiles>c1ccc2cocc2c1</smiles>

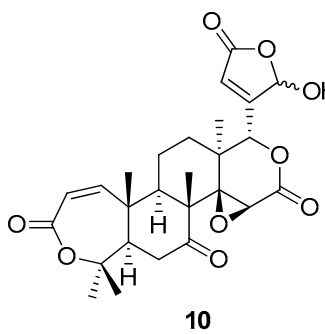

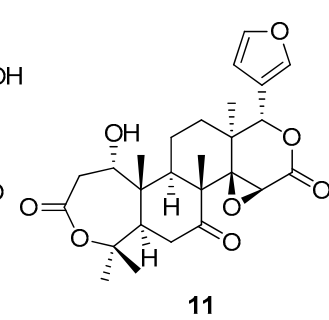

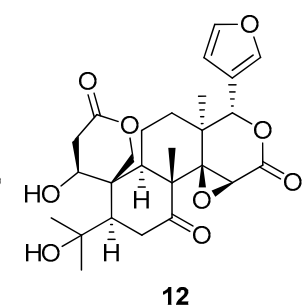

Figure 1. Structures of limonoids isolated from peels of C. reticulata.

21,23-Dihydro-21-hydroxy-23-oxonomilin (1) was obtained as colorless crystals. Its molecular formula was established as $\mathrm{C}_{28} \mathrm{H}_{34} \mathrm{O}_{11}(\mathrm{~m} / z \text { 547.2178 [M + H] }]^{+}$, calcd.: 547.2179) by the HRFABMS. The IR spectrum showed the presence of hydroxy $\left(3466 \mathrm{~cm}^{-1}\right)$ and carbonyl $\left(1733 \mathrm{~cm}^{-1}\right)$ groups. The ${ }^{1} \mathrm{H}$ - and ${ }^{13} \mathrm{C}-\mathrm{NMR}$ spectra indicated the presence of six methyl groups observed as singlets including acetyl methyls $\left(\delta_{\mathrm{H}} 1.11(\mathrm{~s}), 1.16(\mathrm{~s}), 1.34(\mathrm{~s}), 1.48(\mathrm{~s}), 1.56(\mathrm{~s}), 2.10(\mathrm{~s})\right)$, three oxymethines $\left(\delta_{\mathrm{H}} 3.70(\mathrm{~s}), 5.05(\mathrm{brd}), 5.34(\mathrm{brd}) ; \delta_{\mathrm{C}} 52.6(\mathrm{~d}), 70.7(\mathrm{~d}), 78.1(\mathrm{~d})\right)$, two unprotonated oxycarbons $\left(\delta_{\mathrm{C}} 65.0(\mathrm{~s}), 84.4(\mathrm{~s})\right)$, a hemiacetal $\left(\delta_{\mathrm{H}} 5.99(\mathrm{brs}) ; \delta_{\mathrm{C}} 97.4(\mathrm{~d})\right)$, a trisubstituted olefin $\left(\delta_{\mathrm{H}} 6.30(\mathrm{dd})\right.$; $\left.\delta_{C} 123.2(\mathrm{~d}), 162.5(\mathrm{~s})\right)$, a ketone $\left(\delta_{\mathrm{C}} 206.5(\mathrm{~s})\right)$, four ester carbonyls $\left(\delta_{\mathrm{C}} 165.5(\mathrm{~s}), 168.9(\mathrm{~s}), 169.1(\mathrm{~s})\right.$, 169.6 (s)) (Table 1). In the HMBC experiment, the following correlations were observed: Me-18 $\left(\delta_{\mathrm{H}}\right.$ $1.11(\mathrm{~s})) / \mathrm{C}-12, \mathrm{C}-13, \mathrm{C}-14\left(\delta_{\mathrm{C}} 65.0(\mathrm{~s})\right)$, and C-17 $\left(\delta_{\mathrm{C}} 78.1(\mathrm{~d})\right) ; \mathrm{Me}-19\left(\delta_{\mathrm{H}} 1.34(\mathrm{~s})\right) / \mathrm{C}-1\left(\delta_{\mathrm{C}} 70.7(\mathrm{~d})\right)$, C-5, C-9, and C-10; Me-28 ( $\left.\delta_{\mathrm{H}} 1.48(\mathrm{~s})\right)$ and Me-29 $\left(\delta_{\mathrm{H}} 1.56(\mathrm{~s})\right) / \mathrm{C}-4\left(\delta_{\mathrm{C}} 84.4(\mathrm{~s})\right)$ and C-5; Me-30 $\left(\delta_{\mathrm{H}}\right.$ $1.16(\mathrm{~s})) / \mathrm{C}-7\left(\delta_{\mathrm{C}} 206.5(\mathrm{~s})\right), \mathrm{C}-8, \mathrm{C}-9$, and C-14; H-1 $\left(\delta_{\mathrm{H}} 5.05(\mathrm{brd})\right) / \mathrm{C}-3\left(\delta_{\mathrm{C}} 169.1(\mathrm{~s})\right)$ and 1-OCOCH 3 $\left(\delta_{\mathrm{C}} 169.6(\mathrm{~s})\right) ; \mathrm{H}-15\left(\delta_{\mathrm{H}} 3.70(\mathrm{~s})\right) / \mathrm{C}-16\left(\delta_{\mathrm{C}} 165.5(\mathrm{~s})\right) ; \mathrm{H}-22\left(\delta_{\mathrm{H}} 6.30(\mathrm{dd})\right) / \mathrm{C}-21\left(\delta_{\mathrm{C}} 97.4(\mathrm{~d})\right)$ (Figure 2). In addition, a trisubstituted olefin existed at $\mathrm{C}-20$ and $\mathrm{C}-22$, and a hemiactal carbon existed at $\mathrm{C}-21$ since HMBC correlations were observed from H-17 $\left(\delta_{\mathrm{H}} 5.34(\mathrm{brd})\right)$ to the acetal carbon $\left(\delta_{\mathrm{C}} 97.4(\mathrm{~d})\right)$ and olefin carbons $\left(\delta_{C} 123.2(\mathrm{~d}), 162.5(\mathrm{~s})\right)$. The above data suggested that compound 1 had a similar structure to nomilin [22] except for the lack of a furan ring and the presence of a $\beta$-substituted $\gamma$-hydroxybutenolide group. The following correlations were also observed in the ${ }^{1} \mathrm{H}-{ }^{1} \mathrm{H}$ COSY experiment; $\mathrm{H}-1 / \mathrm{H}_{2}-2$; $\mathrm{H}-5 / \mathrm{H}_{2}-6 ; \mathrm{H}-9 / \mathrm{H}_{2}-11 ; \mathrm{H}_{2}-11 / \mathrm{H}_{2}-12$. In the NOESY experiment, the following correlations were observed; Me-28/H-6 $\alpha$; Me-18/H-9 $\alpha, \mathrm{H}-22$, and 1-OCOCH${ }_{3} ; \mathrm{H}-2 \beta / \mathrm{Me}-19$ and Me-29; H-6 $\beta / \mathrm{Me}-19$ and Me-30; H-12 $\beta / \mathrm{H}-17$ (Figure 3). Therefore, the structure of 1 was established, as shown in Figure 1. Compound 1 was an inseparable mixture of C-21 hemiacetal epimers. 
Table 1. ${ }^{1} \mathrm{H}-(600 \mathrm{MHz})$ and ${ }^{13} \mathrm{C}-\mathrm{NMR}(150 \mathrm{MHz})$ spectra data of compounds $\mathbf{1}$ (in $\left.\mathrm{CDCl}_{3}\right), 2$ (in $\left.\left(\mathrm{CD}_{3}\right)_{2} \mathrm{CO}\right)$ and 3 (in $\mathrm{CDCl}_{3}+1$ drop $\mathrm{CD}_{3} \mathrm{OD}$ ) a

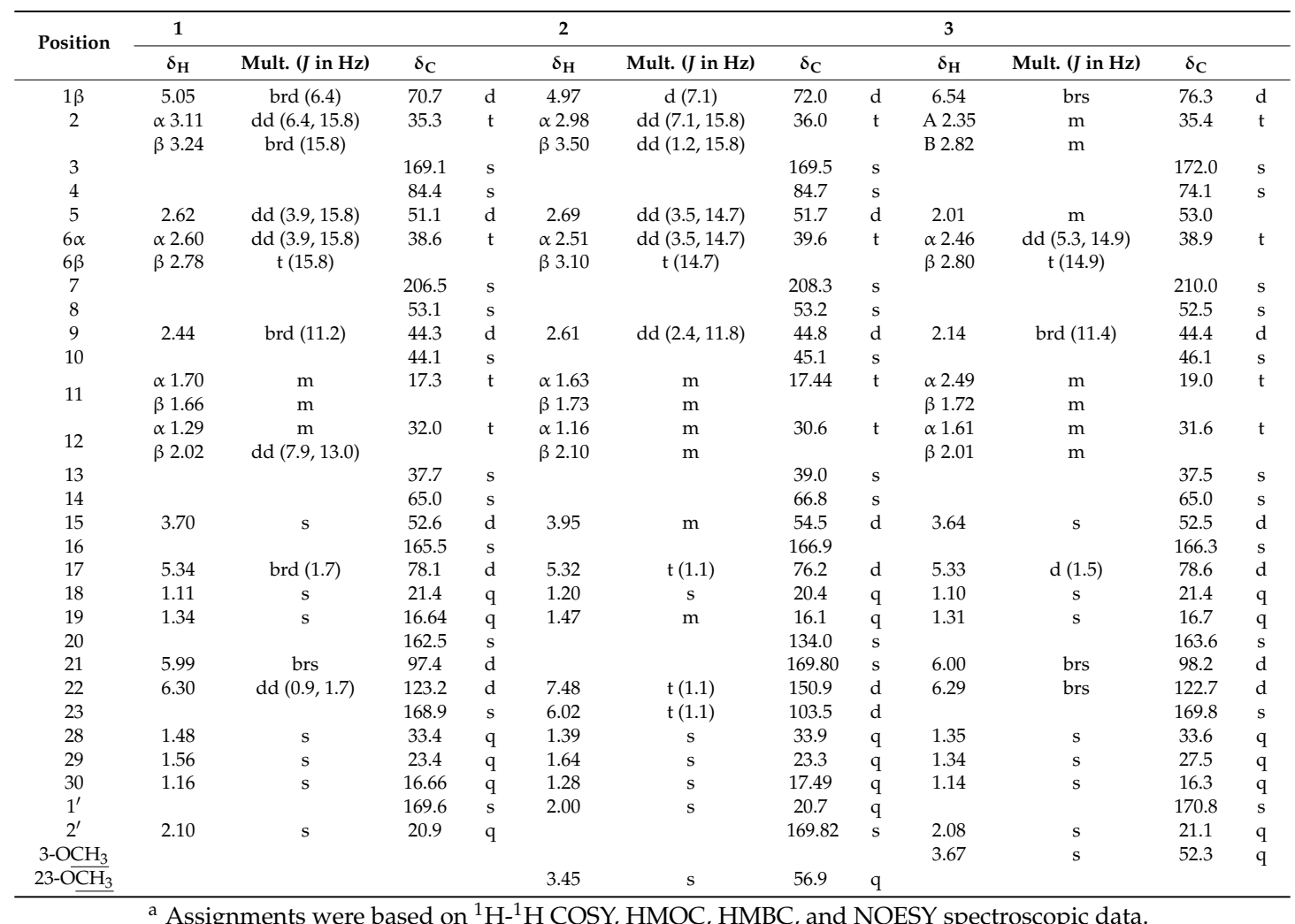

${ }^{\text {a }}$ Assignments were based on ${ }^{1} \mathrm{H}-{ }^{1} \mathrm{H}$ COSY, HMQC, HMBC, and NOESY spectroscopic data.

(A)

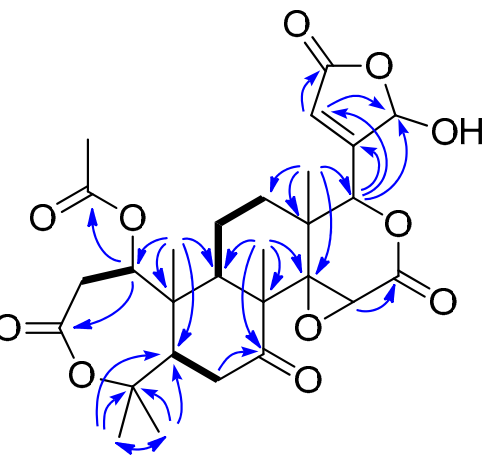

(B)

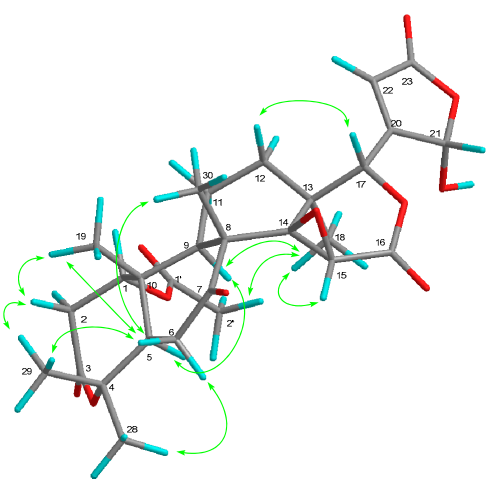

Figure 2. Key $\mathrm{HMBC}(\longrightarrow)$ and ${ }^{1} \mathrm{H}^{-1} \mathrm{H}$ COSY $(\longrightarrow)(\mathbf{A})$ and $\mathrm{NOE}(\longrightarrow-$ ) (B) correlations of compound 1.

21,23-dihydro-23-methoxy-21-oxonomilin (2) was obtained as colorless crystals. Its molecular formula was established as $\mathrm{C}_{29} \mathrm{H}_{32} \mathrm{O}_{11}\left(\mathrm{~m} / z 561.2346[\mathrm{M}+\mathrm{H}]^{+}\right.$, calcd.: 561.2336) by the HRFABMS. Compound 2 was suggested to have a similar structure to 1 except for the absence of a $\beta$-substituted $\gamma$-hydroxybutenolide group and the presence of an $\alpha$-substituted $\gamma$-methoxybutenolide group because of the HMBC correlations from H-17 $\left(\delta_{\mathrm{H}} 5.32(\mathrm{t})\right)$ to an ester carbonyl carbon $\left(\delta_{\mathrm{C}} 169.8(\mathrm{~s})\right)$ and a trisubstituted olefin $\left(\delta_{C} 134.0(\mathrm{~s}), 150.9(\mathrm{~d})\right)$. Therefore, the structure of 2 was established, as shown in Figure 1. The configuration at C-23 was not determined.

21,23-dihydro-21-hydroxy-23-oxonomilinic acid methyl ester (3), an amorphous solid, possessed the molecular formula $\mathrm{C}_{29} \mathrm{H}_{38} \mathrm{O}_{12}\left(\mathrm{~m} / z\right.$ $601.2264[\mathrm{M}+\mathrm{Na}]^{+}$, calcd.: 601.2261). The ${ }^{1} \mathrm{H}-$ and ${ }^{13} \mathrm{C}-\mathrm{NMR}$ spectra showed three methyls observed as singlet including acetyl and methoxy groups $\left(\delta_{\mathrm{H}} 1.10(\mathrm{~s})\right.$, 
$1.14(\mathrm{~s}), 1.31(\mathrm{~s}), 2.08(\mathrm{~s}), 3.67(\mathrm{~s}))$, a hydroxy propyl $\left(\delta_{\mathrm{H}} 1.34(\mathrm{~s}), 1.35(\mathrm{~s}) ; \delta_{\mathrm{C}} 74.1(\mathrm{~s})\right)$, three oxymethine $\left(\delta_{\mathrm{H}} 3.64(\mathrm{~s}), 5.33(\mathrm{~d}), 6.54(\mathrm{brs}) ; \delta_{\mathrm{C}} 52.5(\mathrm{~d}), 76.3(\mathrm{~d}), 78.6(\mathrm{~d})\right)$, a hemiacetal $\left(\delta_{\mathrm{H}} 6.00(\mathrm{brs}) ; \delta_{\mathrm{C}} 98.2(\mathrm{~d})\right)$, an unprotonated oxycarbon $\left(\delta_{C} 65.0(\mathrm{~s})\right)$, a trisubstituted olefin $\left(\delta_{\mathrm{H}} 6.29(\mathrm{brs}) ; \delta_{\mathrm{C}} 122.7(\mathrm{~d}), 163.6(\mathrm{~s})\right)$, a ketone $\left(\delta_{C} 210.0(\mathrm{~s})\right)$, four ester carbonyls $\left(\delta_{C} 166.3(\mathrm{~s}), 169.8(\mathrm{~s}), 170.8(\mathrm{~s}), 172.0(\mathrm{~s})\right)$. In the HMBC experiment, the following correlations were observed: Me-18 $\left(\delta_{\mathrm{H}} 1.10(\mathrm{~s})\right) / \mathrm{C}-12, \mathrm{C}-13$, C-14 $\left(\delta_{\mathrm{C}} 65.0(\mathrm{~s})\right), \mathrm{C}-17\left(\delta_{\mathrm{C}} 78.6(\mathrm{~d})\right)$; Me-19 $\left(\delta_{\mathrm{H}} 1.31(\mathrm{~s})\right) / \mathrm{C}-1\left(\delta_{\mathrm{C}} 76.3(\mathrm{~d})\right), \mathrm{C}-5, \mathrm{C}-9, \mathrm{C}-10 ; \mathrm{Me}-28$ $\left(\delta_{\mathrm{H}} 1.35(\mathrm{~s})\right)$ and Me-29 $\left(\delta_{\mathrm{H}} 1.34(\mathrm{~s})\right) / \mathrm{C}-4\left(\delta_{\mathrm{C}} 74.1(\mathrm{~s})\right), \mathrm{C}-5 ; \mathrm{Me}-30\left(\delta_{\mathrm{H}} 1.14(\mathrm{~s})\right) / \mathrm{C}-7\left(\delta_{\mathrm{C}} 210.0(\mathrm{~s})\right)$, C-8, C-9, C-14; H-2B $\left(\delta_{\mathrm{H}} 2.82(\mathrm{~m})\right), 3-\mathrm{OCH}_{3}\left(\delta_{\mathrm{H}} 3.67(\mathrm{~s})\right) / \mathrm{C}-3\left(\delta_{\mathrm{C}} 172.0(\mathrm{~s})\right) ; \mathrm{H}-15\left(\delta_{\mathrm{H}} 3.64(\mathrm{~s})\right) / \mathrm{C}-16$

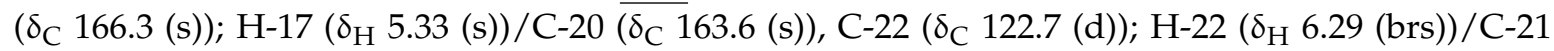
$\left(\delta_{C} 98.2(\mathrm{~d})\right), \mathrm{C}-23\left(\delta_{\mathrm{C}} 169.8(\mathrm{~s})\right)$. The following correlations were also observed in the ${ }^{1} \mathrm{H}_{-}{ }^{1} \mathrm{H}$ COSY experiment: $\mathrm{H}-1\left(\delta_{\mathrm{H}} 6.54\right.$ (brs))-H-2A; H-5- $\mathrm{H}_{2}-6 ; \mathrm{H}-9-\mathrm{H}_{2}-11-\mathrm{H}_{2}-12$. From the above data, compound 3 was similar to 3-O-methyl 21,23-dihydro-23-hydroxy-21-oxonomilinic acid [23] except for the absence of an $\alpha$-substituted $\gamma$-hydroxybutenolide group and the presence of a $\beta$-substituted $\gamma$-hydroxybutenolide group. The relative configuration was determined by the NOESY experiment (Figure 3). The configuration of $\mathrm{C}-1$ was determined as $S$ because of the following NOE correlations: $\mathrm{H}-1\left(\delta_{\mathrm{H}} 6.54(\mathrm{brs})\right) / \mathrm{H}-2 \mathrm{~B}\left(\delta_{\mathrm{H}} 2.82(\mathrm{~m})\right), \mathrm{Me}-19\left(\delta_{\mathrm{H}} 1.31(\mathrm{~s})\right), \mathrm{Me}-28\left(\delta_{\mathrm{H}} 1.35(\mathrm{~s})\right)$, and Me-29 $\left(\delta_{\mathrm{H}} 1.34(\mathrm{~s})\right)$; $\mathrm{H}-2 \mathrm{~A}\left(\delta_{\mathrm{H}} 2.35(\mathrm{~m})\right) / \mathrm{H}-9\left(\delta_{\mathrm{H}} 2.14(\mathrm{brd})\right)$; Me-19/Me-2' $\left(\delta_{\mathrm{H}} 3.67(\mathrm{~s})\right)$. Therefore, the structure of 3 was established as shown in Figure 1. Compound 3 was also an inseparable mixture of C-21 hemiacetal epimers.

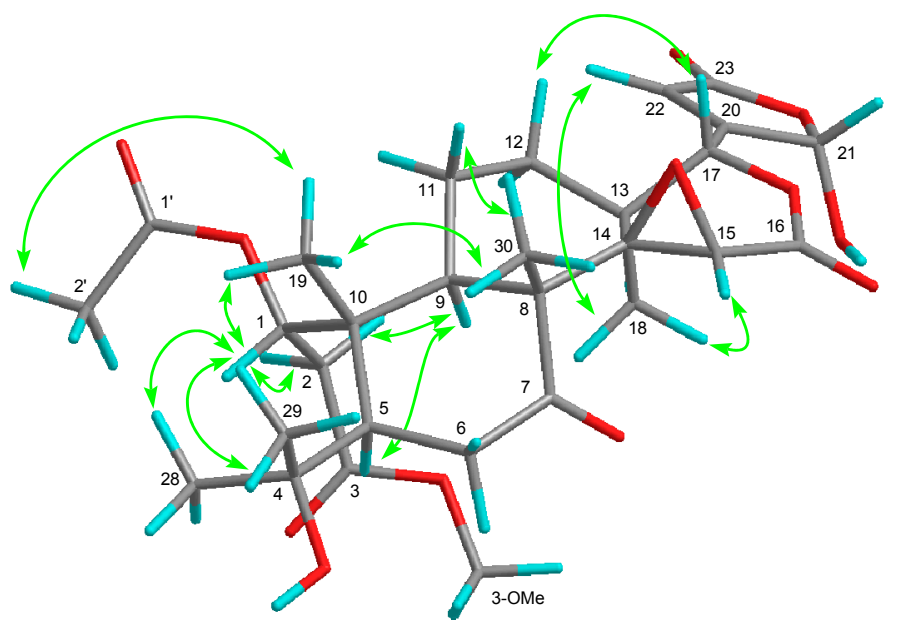

Figure 3. Key NOE correlations $(\longrightarrow$ ) of compound 3.

21,23-dihydro-23-methoxy-21-oxolimonin (4) was obtained as an amorphous solid. Its molecular formula was established as $\mathrm{C}_{27} \mathrm{H}_{32} \mathrm{O}_{10}\left(\mathrm{~m} / z 539.1891[\mathrm{M}+\mathrm{Na}]^{+}\right.$, calcd.: 539.1894$)$ by the HRFABMS. The absorbance in the IR spectrum indicated hydroxy $\left(3434 \mathrm{~cm}^{-1}\right)$ and carbonyl $\left(1750 \mathrm{~cm}^{-1}\right)$ groups. The ${ }^{1} \mathrm{H}$ - and ${ }^{13} \mathrm{C}-\mathrm{NMR}$ spectra indicated five methyl groups observed as singlets including methoxy group $\left(\delta_{\mathrm{H}} 1.09(\mathrm{~s}), 1.18(\mathrm{~s}), 1.18(\mathrm{~s}), 1.29(\mathrm{~s}), 3.60(\mathrm{~s})\right)$, an oxymethylene $\left(\delta_{\mathrm{H}} 4.46(\mathrm{~d}), 4.74(\mathrm{~d})\right.$; $\left.\delta_{\mathrm{C}} 65.1(\mathrm{t})\right)$ three oxymethines $\left(\delta_{\mathrm{H}} 4.03(\mathrm{brd}), 4.12(\mathrm{~s}), 5.43(\mathrm{t}) ; \delta_{\mathrm{C}} 53.8(\mathrm{~d}), 75.2(\mathrm{~d}), 79.2(\mathrm{~d})\right)$, two unprotonated oxycarbons $\left(\delta_{\mathrm{C}} 65.7(\mathrm{~s}), 80.3(\mathrm{~s})\right)$, an acetal $\left(\delta_{\mathrm{H}} 5.77(\mathrm{t}) ; \delta_{\mathrm{C}} 102.5(\mathrm{~d})\right)$ a trisubstituted olefin $\left(\delta_{\mathrm{H}} 7.25(\mathrm{t}) ; \delta_{\mathrm{C}} 133.8(\mathrm{~s}), 149.1(\mathrm{~d})\right)$, a ketone $\left(\delta_{\mathrm{C}} 206.1(\mathrm{~s})\right)$, and three ester carbonyls $\left(\delta_{\mathrm{C}} 166.0(\mathrm{~s})\right.$, 168.8 (s), 168.9 (s)) (Table 2). The HMBC and ${ }^{1} \mathrm{H}^{-1} \mathrm{H}$ COSY correlations (Figure 4) suggested that compound 4 had a similar structure to limonin [15] except for the lack of a furan ring and presence of an $\alpha$-substituted $\gamma$-methoxybutenolide group. In the NOESY experiment, the following correlations

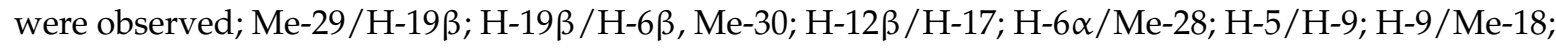
Me-18/23-OMe; Me-30/H-19 $\alpha$; H-19 $\alpha / \mathrm{H}-2 \alpha$ (Figure 4). Therefore, the structure of 4 was established, as shown in Figure 1. The configuration at C-23 was not established. 
(A)

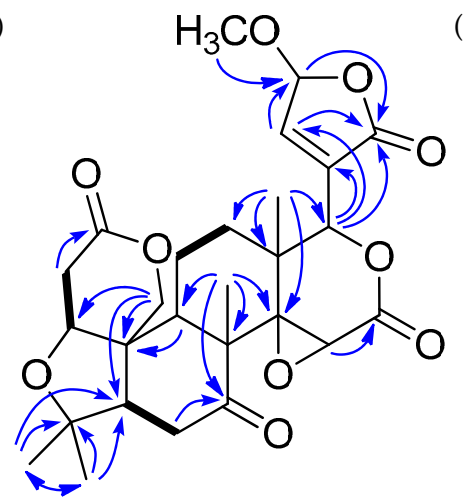

(B)

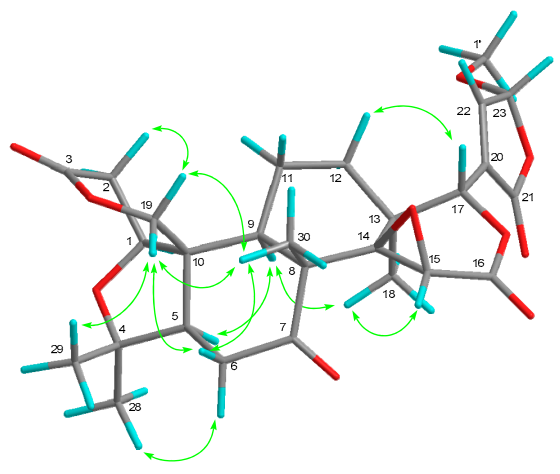

Figure 4. Key $\mathrm{HMBC}(\longrightarrow)$ and ${ }^{1} \mathrm{H}^{-1} \mathrm{H}$ COSY $(\longrightarrow)(\mathbf{A})$ and $\mathrm{NOE}(\longrightarrow)(\mathbf{B})$ correlations of compound 4.

Table 2. ${ }^{1} \mathrm{H}-(600 \mathrm{MHz})$ and ${ }^{13} \mathrm{C}-\mathrm{NMR}(150 \mathrm{MHz})$ spectra data of compounds 4 (in $\left.\mathrm{CDCl}_{3}\right)$ and 5 (in $\left.\left(\mathrm{CD}_{3}\right)_{2} \mathrm{CO}\right)^{\text {a }}$.

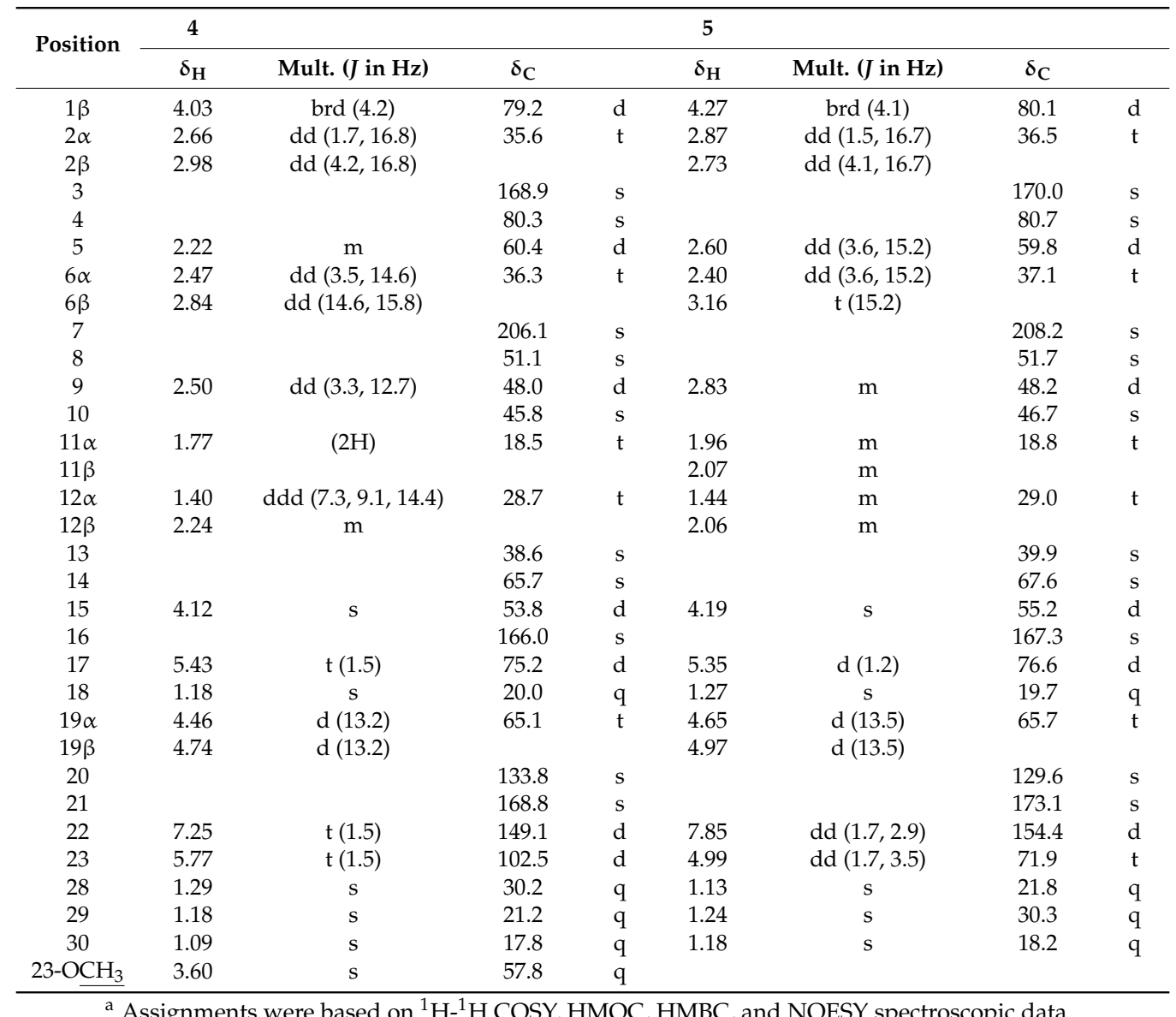

21,23-Dihydro-21-oxolimonin (5) was obtained as an amorphous solid. Its molecular formula was established as $\mathrm{C}_{26} \mathrm{H}_{30} \mathrm{O}_{9}\left(\mathrm{~m} / z 487.1967[\mathrm{M}+\mathrm{H}]^{+}\right.$, calcd.: 487.1968). Compound 5 was similar to 4 except for the absence of an acetal carbon and the presence of an oxymethylene at $\mathrm{C}-23$. The structure was confirmed by 2D NMR spectra including HSQC, HMBC, ${ }^{1} \mathrm{H}-{ }^{1} \mathrm{H}$ COSY, and NOESY experiments. Therefore, the structure of $\mathbf{5}$ was established, as shown in Figure 1.

Macrophages may be a potential therapeutic target for inflammatory diseases [24]. Activated macrophages release pro-inflammatory mediators, such as NO, reactive oxygen species, interleukin- $1 \beta$, 
tumor necrosis factor- $\alpha$, and other inflammatory mediators, which play important roles in biological defense. However, the overexpression of these mediators had been implicated in diseases such as osteoarthritis, rheumatoid arthritis, and diabetes because the increased production of pro-inflammatory mediators induces severe or chronic inflammation [24]. Isolated compounds (1-12), and $N^{\mathrm{G}}$. monomethyl-L-arginine, acetate (L-NMMA), which is a NO synthase inhibitor and was used as a positive control, were evaluated for macrophage activation by the inhibitory assay of NO production in RAW264.7 mouse macrophages stimulated by lipopolysaccharide (LPS). Compounds 1-7, and 9-12 did not exhibit cytotoxicity at 1-30 $\mu \mathrm{M}$ (Table 3). Of these, compound 2 ( $\left.\mathrm{IC}_{50} 25.4 \mu \mathrm{M}\right)$ showed a comparable inhibitory effect on NO production to L-NMMA ( $\left.\mathrm{IC}_{50} 23.9 \mu \mathrm{M}\right)$ (Table 3). Compounds 3, 5, 8, 11 and 12 showed some inhibitory activities (produced NO ratio 3: 80.3\%; 5: 83.2\%; 8: 76.0\%; 11: $78.1 \% ; 12: 73.9 \%$ at $30 \mu \mathrm{M}$ ). The other limonoids did not exhibit inhibitory effects on NO production. These results suggested that compound 2 has potential as an anti-inflammatory disease agent.

Table 3. Inhibitory effects of NO production by limonoids from peels of Citrus reticulata.

\begin{tabular}{|c|c|c|c|c|c|}
\hline \multicolumn{6}{|c|}{ Inhibitory Ratio of NO \% (Cell Viability \%) ${ }^{a, b}$} \\
\hline Compound & $1 \mu \mathrm{M}$ & $3 \mu \mathrm{M}$ & $10 \mu \mathrm{M}$ & $30 \mu \mathrm{M}$ & $\mathrm{IC}_{50}(\mu \mathrm{M})$ \\
\hline 1 & $\begin{array}{l}109.9 \pm 2.8 \\
(99.1 \pm 1.3)\end{array}$ & $\begin{array}{c}110.9 \pm 1.7 \\
(102.5 \pm 1.8)\end{array}$ & $\begin{array}{c}104.8 \pm 2.2 \\
(103.7 \pm 0.7)\end{array}$ & $\begin{array}{l}106.6 \pm 2.5 \\
(99.3 \pm 1.3)\end{array}$ & $>30$ \\
\hline 2 & $\begin{array}{c}95.2 \pm 1.6 \\
(101.3 \pm 1.5)\end{array}$ & $\begin{array}{l}89.8 \pm 2.1 * \\
(98.7 \pm 1.4)\end{array}$ & $\begin{array}{c}80.4 \pm 2.1 * * \\
(99.1 \pm 0.3)\end{array}$ & $\begin{array}{c}39.3 \pm 0.5^{* *} \\
(94.5 \pm 0.4)\end{array}$ & 25.4 \\
\hline 3 & $\begin{array}{c}99.4 \pm 0.8 \\
(96.8 \pm 0.2)\end{array}$ & $\begin{array}{c}99.4 \pm 2.3 \\
(94.5 \pm 0.3)\end{array}$ & $\begin{array}{c}94.1 \pm 1.9 \\
(93.2 \pm 0.4)\end{array}$ & $\begin{array}{c}80.3 \pm 1.2^{* *} \\
(93.4 \pm 0.6)\end{array}$ & $>30$ \\
\hline 4 & $\begin{array}{c}96.2 \pm 7.9 \\
(102.6 \pm 0.4)\end{array}$ & $\begin{array}{c}94.0 \pm 2.7 \\
(97.0 \pm 0.3)\end{array}$ & $\begin{array}{l}100.8 \pm 1.2 \\
(95.1 \pm 0.2)\end{array}$ & $\begin{array}{c}95.2 \pm 3.4 \\
(91.7 \pm 0.5)\end{array}$ & $>30$ \\
\hline 5 & $\begin{array}{c}99.5 \pm 5.6 \\
(97.9 \pm 1.9)\end{array}$ & $\begin{array}{l}100.6 \pm 4.7 \\
(97.9 \pm 0.2)\end{array}$ & $\begin{array}{c}93.8 \pm 1.6 \\
(99.5 \pm 0.5)\end{array}$ & $\begin{array}{l}83.2 \pm 5.6^{*} \\
(96.6 \pm 0.2)\end{array}$ & $>30$ \\
\hline 6 & $\begin{array}{c}102.3 \pm 4.4 \\
(100.1 \pm 0.3)\end{array}$ & $\begin{array}{c}93.4 \pm 5.7 \\
(95.6 \pm 0.5)\end{array}$ & $\begin{array}{c}90.9 \pm 3.1 \\
(97.2 \pm 0.9)\end{array}$ & $\begin{array}{c}86.0 \pm 5.5 \\
(99.3 \pm 0.9)\end{array}$ & $>30$ \\
\hline 7 & $\begin{array}{c}99.8 \pm 1.4 \\
(102.3 \pm 1.5)\end{array}$ & $\begin{array}{c}99.0 \pm 3.0 \\
(106.3 \pm 2.2)\end{array}$ & $\begin{array}{c}96.8 \pm 2.5 \\
(104.2 \pm 1.4)\end{array}$ & $\begin{array}{c}93.9 \pm 4.0 \\
(100.2 \pm 1.4)\end{array}$ & $>30$ \\
\hline 8 & $\begin{array}{c}97.2 \pm 1.1 \\
(98.1 \pm 2.4)\end{array}$ & $\begin{array}{c}94.7 \pm 2.4 \\
(97.6 \pm 0.7)\end{array}$ & $\begin{array}{c}90.9 \pm 1.6^{* *} \\
(92.0 \pm 0.5)\end{array}$ & $\begin{array}{c}76.0 \pm 2.2^{* *} \\
(89.3 \pm 0.5)\end{array}$ & $>30$ \\
\hline 9 & $\begin{array}{c}94.3 \pm 4.3 \\
(101.4 \pm 0.7)\end{array}$ & $\begin{array}{c}85.2 \pm 6.4 \\
(97.7 \pm 0.6)\end{array}$ & $\begin{array}{c}91.3 \pm 2.8 \\
(101.3 \pm 1.2)\end{array}$ & $\begin{array}{c}89.9 \pm 3.2 \\
(97.6 \pm 2.1)\end{array}$ & $>30$ \\
\hline 10 & $\begin{array}{c}95.0 \pm 4.2 \\
(104.7 \pm 2.3)\end{array}$ & $\begin{array}{c}96.8 \pm 1.3 \\
(101.8 \pm 1.0)\end{array}$ & $\begin{array}{c}97.7 \pm 2.2 \\
(98.9 \pm 0.5)\end{array}$ & $\begin{array}{c}86.6 \pm 1.6 \\
(98.6 \pm 1.8)\end{array}$ & $>30$ \\
\hline 11 & $\begin{array}{l}103.6 \pm 3.1 \\
(95.9 \pm 0.1)\end{array}$ & $\begin{array}{c}93.2 \pm 3.2 \\
(100.1 \pm 0.4)\end{array}$ & $\begin{array}{l}84.7 \pm 3.2 * \\
(99.3 \pm 1.5)\end{array}$ & $\begin{array}{l}78.1 \pm 3.4 \\
(100.4 \pm 0.6)\end{array}$ & $>30$ \\
\hline 12 & $\begin{array}{c}106.6 \pm 4.1 \\
(100.7 \pm 0.3)\end{array}$ & $\begin{array}{c}92.6 \pm 3.2 \\
(98.1 \pm 1.0)\end{array}$ & $\begin{array}{c}88.4 \pm 5.5 \\
(98.3 \pm 1.6)\end{array}$ & $\begin{array}{c}73.9 \pm 3.4^{* *} \\
(98.3 \pm 1.2)\end{array}$ & $>30$ \\
\hline L-NMMA $^{c}$ & $\begin{array}{c}93.3 \pm 2.2 \\
(101.5 \pm 0.9)\end{array}$ & $\begin{array}{c}91.4 \pm 0.8 \\
(101.9 \pm 0.4)\end{array}$ & $\begin{array}{c}68.9 \pm 4.5^{* *} \\
(98.5 \pm 0.9)\end{array}$ & $\begin{array}{l}43.1 \pm 1.1^{* *} \\
(109.4 \pm 0.5)\end{array}$ & 23.9 \\
\hline
\end{tabular}

a Each value represents the mean \pm standard error (S.E.) of four determinations; ${ }^{b}$ Significant differences from the vehicle control group shown as ${ }^{*} p<0.05$ and ${ }^{* *} p<0.01$; ${ }^{\mathrm{c}}$ Positive control.

\section{Experimental}

\subsection{General Experimental Procedure}

The following chemicals and reagents were purchased: fetal bovine serum (FBS) from Invitrogen Co. (Carlsbad, CA, USA), 3-(4,5-dimethyl-2-thiazolyl)-2,5-diphenyl-2H-tetrazolium bromide (MTT) from Sigma-Aldrich Japan Co. (Tokyo, Japan), Dulbecco's modified Eagle's medium (D-MEM), and antibiotics from Nacalai Tesque, Inc. (Kyoto, Japan). All other chemicals and reagents were of analytical grade. Melting points were determined on a Yanagimoto micro-melting point apparatus and were uncorrected. 
Optical rotations were measured with a JASCO DIP-1000 digital polarimeter. IR spectra were recorded on a Perkin-Elmer 1720X FTIR spectrophotometer. UV spectra were measured on a HITACHI U-2000 spectrometer. ${ }^{1} \mathrm{H}-(600 \mathrm{MHz})$ and ${ }^{13} \mathrm{C}-(150 \mathrm{MHz}) \mathrm{NMR}$ spectra were recorded on an Agilent vnmrs600 with tetramethylsilane as the internal standard. HR-FAB-MS was recorded on a JEOL JMS-7000 mass spectrometer. Silica gel (70-230 mesh, Merck) and silica gel 60 (230-400 mesh, Nacalai tesque, Inc., Kyoto, Japan) were used for column chromatography and medium-pressure liquid chromatography, respectively. HPLC was carried out on an ODS column (Cosmosil 5C $C_{18}-M S$-II column (Nacalai Tesque, Inc., Kyoto, Japan), $25 \mathrm{~cm} \times 20 \mathrm{~mm}$ i.d., $5 \mu \mathrm{m}$ particle size) at $35^{\circ} \mathrm{C}$ with $\mathrm{MeCN} / \mathrm{H}_{2} \mathrm{O}(1: 1$ (System I), 45:55 (System III), 2:3 (System IV), 3:7 (System V), flow rate $4.0 \mathrm{~mL} / \mathrm{min}$ ), Cosmosil PAQ (Nacalai Tesque, Inc., Kyoto, Japan), $25 \mathrm{~cm} \times 20 \mathrm{~mm}$ i.d., $5 \mu \mathrm{m}$ particle size) at $35^{\circ} \mathrm{C}\left(\mathrm{MeCN} / \mathrm{H}_{2} \mathrm{O}\right.$, flow rate $4.0 \mathrm{~mL} / \mathrm{min}$, (1:1) (System $I I))$.

\subsection{Plant Material}

The fruits of C. reticulata were produced in Wakayama prefecture (Japan) in 2013. A voucher specimen of the peels of Citrus reticulata was deposited in the Herbarium of the Laboratory of Medicinal Chemistry, Osaka University of Pharmaceutical Sciences.

\subsection{Extraction and Isolation}

The peels of Citrus reticulata fruits (dry weight $4952 \mathrm{~g}$ ), produced in Wakayama, were subjected to extraction with $\mathrm{MeOH}$ under reflux ( $15 \mathrm{~L}, 3$ days, 3 times). The $\mathrm{MeOH}$ extract $(280 \mathrm{~g})$ was then partitioned between AcOEt and $\mathrm{H}_{2} \mathrm{O}(9 \mathrm{~L} / 9 \mathrm{~L}, 4$ times). The AcOEt-soluble fraction $(280 \mathrm{~g})$ was subjected to $\mathrm{SiO}_{2}$ column chromatography (CC) $\left(\mathrm{SiO}_{2}\right.$ (3.5 kg); hexane/AcOEt (5:1, 1:1, and 0:1), and AcOEt:MeOH (1:1, and 0:1) in increasing order of polarity) resulting in twenty fractions (Fr. A-T).

Fr. J (11 g), eluted with AcOEt, was subjected to $\mathrm{SiO}_{2} \mathrm{CC}$ to yield 10 fractions $\left(\mathrm{SiO}_{2}(300 \mathrm{~g})\right.$; hexane/AcOEt (5:1, 3:1, 1:1, 1:5, 1:10, 1:20, and 0:1), and AcOEt:MeOH (5:1, and 0:1) in increasing order of polarity), J1-J10. Preparative HPLC (System III) of J6 (237 mg), eluted with hexane/AcOEt (1:10), gave 5 ( $4.5 \mathrm{mg}$; $\left.\mathrm{t}_{\mathrm{R}} 24.6 \mathrm{~min}\right)$.

Fr. K (11 g), eluted with AcOEt, was subjected to $\mathrm{SiO}_{2} \mathrm{CC}$ to yield 11 fractions $\left(\mathrm{SiO}_{2}(270 \mathrm{~g})\right.$; hexane/AcOEt (1:1 and 0:1), and AcOEt:MeOH (1:1, and 0:1) in increasing order of polarity), K1-K11, followed by $\mathrm{SiO}_{2} \mathrm{CC}$ of $\mathrm{K} 5(1 \mathrm{~g})$, eluted with AcOEt, to yield 8 fractions $\left(\mathrm{SiO}_{2}(60 \mathrm{~g}) ;\right.$ AcOEt and $\mathrm{MeOH}), \mathrm{K} 5-1-\mathrm{K} 5-8$. Preparative HPLC (System IV) of K5-2 (215 mg), eluted with AcOEt, gave 12 fractions, K5-2-1-K5-2-12. K5-2-9 was identified as 6 ( 26 mg; $\mathrm{t}_{\mathrm{R}} 47.4 \mathrm{~min}$ ). Preparative HPLC (System $V$ ) of K5-2-2 (11 mg) gave 7 (5.4 mg; $\mathrm{t}_{\mathrm{R}} 47.7 \mathrm{~min}$ ). Preparative HPLC (System $V$ ) of K5-2-3 (20 mg) gave 8 $\left(3.0 \mathrm{mg} ; \mathrm{t}_{\mathrm{R}} 58.6 \mathrm{~min}\right.$ ) and 9 (1.3 mg; $\left.\mathrm{t}_{\mathrm{R}} 49.0 \mathrm{~min}\right)$. Preparative HPLC (System $V$ ) of K5-2-6 (10 mg) gave $10\left(1.9 \mathrm{mg} ; \mathrm{t}_{\mathrm{R}} 91.8 \mathrm{~min}\right)$. Preparative HPLC (System $V$ ) of K5-2-8 $\left(7.9 \mathrm{mg}\right.$ ) gave 3 (1.1 mg; $\mathrm{t}_{\mathrm{R}} 92.0 \mathrm{~min}$ ).

Preparative HPLC (System IV) of K5-3 (397 mg) gave 8 (3.7 mg; $\mathrm{t}_{\mathrm{R}} 58.8 \mathrm{~min}$ ). Fr. K6 (3 g), eluted with AcOEt, was subjected to $\mathrm{SiO}_{2} \mathrm{CC}$, to yield 8 fractions $\left(\mathrm{SiO}_{2}(60 \mathrm{~g})\right.$; AcOEt and $\left.\mathrm{MeOH}\right), \mathrm{K} 6-1-\mathrm{K} 6-8$. Preparative HPLC (System I) of K6-2 (376 mg), eluted with AcOEt, gave 10 fractions, K6-2-1-K6-2-10. Preparative HPLC (System V) of K6-2-1 (135 mg) gave $\mathbf{1}\left(14 \mathrm{mg} ; \mathrm{t}_{\mathrm{R}} 104.5 \mathrm{~min}\right)$ and $\mathbf{2}\left(2.4 \mathrm{mg} ; \mathrm{t}_{\mathrm{R}} 186.8\right.$ min). Preparative HPLC (System IV) of K6-2-3 (4.2 mg) gave 4 ( $2.5 \mathrm{mg}$; $\mathrm{t}_{\mathrm{R}} 51.6 \mathrm{~min}$ ).Preparative HPLC (System II) of K6-2-3 (1120 mg), eluted with AcOEt, gave 15 fractions, K6-3-1-K6-3-15. K6-3-9 was identified as 11 (42 mg; $\mathrm{t}_{\mathrm{R}} 27.8 \mathrm{~min}$ ). Preparative HPLC (System $V$ ) of K6-3-4 (59 mg) gave $\mathbf{1}(12 \mathrm{mg}$ ) and 12 (5.5 $\left.\mathrm{mg} ; \mathrm{t}_{\mathrm{R}} 92.6 \mathrm{~min}\right)$.

21,23-Dihydro-21-hydroxy-23-oxonomilin (1). Colorless crystals, m.p. 200-201 ${ }^{\circ} \mathrm{C} ;[\alpha]_{\mathrm{D}}^{21}-100.0(c=0.083$, $\mathrm{EtOH}) ; \mathrm{IR} v_{\max }{ }^{\mathrm{KBr}} \mathrm{cm}^{-1}: 3466,2921,2852,1747,1733,1717,1596,1378,1232,1025$; UV $\lambda_{\max }{ }^{\mathrm{EtOH}} \mathrm{nm}$ (loge): 211.5 (3.82); FAB-MS $m / z: 547[\mathrm{M}+\mathrm{H}]^{+}, 569$ [M + Na] ${ }^{+}$; HR-FAB-MS $m / z: 547.2178$ (calcd. for 547.2179: $\left.\mathrm{C}_{28} \mathrm{H}_{35} \mathrm{O}_{11}\right)$.

21,23-Dihydro-23-methoxy-21-oxonomilin (2). Colorless crystals, m.p. $208-210^{\circ} \mathrm{C} ;[\alpha]_{\mathrm{D}}^{12}-15.7(c=0.075$, $\mathrm{EtOH}) ; \mathrm{IR} v_{\max }{ }^{\mathrm{KBr}} \mathrm{cm}^{-1}: 3425,2925,1751,1716,1374,1120,1027 ; \mathrm{UV} \lambda_{\max }{ }^{\mathrm{EtOH}} \mathrm{nm}(\log \varepsilon): 212.5$ 
(3.72); FAB-MS $m / z: 561[\mathrm{M}+\mathrm{H}]^{+}, 583[\mathrm{M}+\mathrm{Na}]^{+}$; HR-FAB-MS $m / z: 561.2346$ (calcd. for 561.2336: $\left.\mathrm{C}_{29} \mathrm{H}_{37} \mathrm{O}_{11}\right)$.

21,23-Dihydro-21-hydroxy-23-oxonomilinic acid methyl ester (3). Amorphous solid; $[\alpha]_{\mathrm{D}}^{25}-54.7$ (c=0.16, EtOH); IR $\gamma_{\max }{ }^{\mathrm{KBr}} \mathrm{cm}^{-1}: 3453,2924,2853,1743,1596,1442,1382,1261,1028 ; \mathrm{UV} \lambda_{\max }{ }^{\mathrm{EtOH}} \mathrm{nm}$ (loge): 203.5 (3.80); FAB-MS m/z: 601 [M + Na] ${ }^{+}$; HR-FAB-MS m/z: 601.2264 (calcd. for 601.2261: $\mathrm{C}_{29} \mathrm{H}_{38} \mathrm{NaO}_{12}$ ).

21,23-Dihydro-23-methoxy-21-oxolimonin (4). Amorphous solid; $[\alpha]_{\mathrm{D}}^{23}-15.7(c=0.12, \mathrm{EtOH})$; IR $\gamma_{\max } \mathrm{KBr}$ $\mathrm{cm}^{-1}$ : 3434, 2922, 2852, 1750, 1713, 1632, 1456, 1384, 1034; UV $\lambda_{\max }{ }^{\mathrm{EtOH}} \mathrm{nm}(\log \varepsilon)$ : 206.5 (3.43); FAB-MS $m / z: 539[\mathrm{M}+\mathrm{Na}]^{+}$; HR-FAB-MS m/z: 539.1891 (calcd. for 539.1894: $\mathrm{C}_{27} \mathrm{H}_{32} \mathrm{NaO}_{10}$ ).

21,23-Dihydro-21-oxolimonin (5). Amorphous solid; $[\alpha]_{\mathrm{D}}^{25}-53.9(c=0.10, \mathrm{EtOH}) ; \mathrm{IR} v_{\max }{ }^{\mathrm{KBr}} \mathrm{cm}^{-1}: 2969$, $1749,1712,1457,1361,1265,1095$; UV $v_{\max }{ }^{\mathrm{EtOH}} \mathrm{nm}(\log \varepsilon): 206.0(3.76)$; FAB-MS $m / z: 487[\mathrm{M}+\mathrm{H}]^{+}$; HR-FAB-MS m/z: 487.1967 (calcd. for 487.1968: $\mathrm{C}_{26} \mathrm{H}_{31} \mathrm{O}_{9}$ ).

\subsection{Cell Culture}

RAW264.7 cells were grown in D-MEM medium supplemented with 10\% FBS and antibiotics (100 units $/ \mathrm{mL}$ penicillin $\mathrm{G}$ sodium salt and $100 \mu \mathrm{g} / \mathrm{mL}$ streptomycin sulfate). Cells were incubated at $37^{\circ} \mathrm{C}$ in a $5 \% \mathrm{CO}_{2}$ humidified incubator.

\subsection{Cytotoxicity Assay}

Cytotoxicity assay was performed according to a method reported previously [25] Briefly, RAW264.7 cells $\left(5 \times 10^{4}\right.$ cells in $\left.100 \mu \mathrm{L}\right)$ were seeded onto a 96-well microplate and incubated for $24 \mathrm{~h}$. DMEM containing test samples (100 $\mu \mathrm{L}$ total volume, final concentration of $30,10,3$, or $1 \mu \mathrm{M})$ dissolved in DMSO (final concentration $0.2 \%$ ) was added. After treatment for $24 \mathrm{~h}$, MTT solution was added. After a 3-h incubation, $20 \%$ sodium dodecyl sulfate in $0.1 \mathrm{M} \mathrm{HCl}$ was added to dissolve the formazan produced in the cells. The absorbance of each well was read at $570 \mathrm{~nm}$ using a microplate reader. The optical density of vehicle control cells was assumed to be $100 \%$.

\subsection{Inhibitory Assay of NO Production}

An inhibitory assay of NO production was performed according to a method reported previously [26] with slight modifications. Briefly, RAW264.7 cells $\left(5 \times 10^{4}\right.$ cells in $\left.100 \mu \mathrm{L}\right)$ were seeded into a 96-well microplate and incubated for $24 \mathrm{~h}$. DMEM containing test samples (100 $\mu \mathrm{L}$ total volume, final concentration of 30,10,3, or $1 \mu \mathrm{M}$ ) dissolved in DMSO (final concentration $0.2 \%$ ), and LPS (final concentration of $5 \mu \mathrm{g} / \mathrm{mL}$ ), was added. After treatment for $24 \mathrm{~h}$, the supernatant of culture medium was transferred to another 96-well microplate, and then $50 \mu \mathrm{L}$ of $0.15 \% \mathrm{~N}$-(1-naphtyl)ethylenediamine in $\mathrm{H}_{2} \mathrm{O}$, and $1.5 \%$ sulfanilamide in $7.5 \%$ phosphoric acid were added. After incubation for $30 \mathrm{~min}$, the absorbance of each well was read at $570 \mathrm{~nm}$ using a microplate reader. The optical density of vehicle control cells was assumed to be $100 \%$.

\section{Conclusions}

In this study, we isolated five new compounds and elucidated their structures. They were limonoids having $\beta$-substituted $\gamma$-hydroxybutenolide $(1,3), \alpha$-substituted $\gamma$-methoxybutenolide $(2,4)$, and $\alpha$-substituted butenolide (5) groups. Compounds $\mathbf{1}$ and $\mathbf{3}$ were inseparable mixtures of C-21 hemiacetal epimers. In NO inhibitory assay, compound $2\left(\mathrm{IC}_{50} 25.4 \mu \mathrm{M}\right)$ possessed a comparable inhibitory effect on NO production to L-NMMA $\left(\mathrm{IC}_{50} 23.9 \mu \mathrm{M}\right)$ without cytotoxicities. These results suggested that compound $\mathbf{2}$ has potential as an anti-inflammatory disease agent.

Supplementary Materials: Supplementary materials are available online.

Acknowledgments: We are grateful to M. Fujitake and K. Minoura of this university for MS and NMR measurements. 
Author Contributions: T.K., T.Y. and R.T. conceived and designed the experiments; T.K., Y.U., Y.H., C.F, T.F. and R.T. performed the experiments; T.K., Y.U., Y.H. and C.F analyzed the data; T.K. wrote the paper.

Conflicts of Interest: The authors declare no conflict of interest.

\section{References}

1. Okada, M.; Mitsuhashi, H. (Eds.) Newly Revised Illustrated Medicinal Plants of the World; Hokuryukan Co., Ltd.: Tokyo, Japan, 2002; p. 249.

2. Sawabe, A.; Morita, M.; Kiso, T.; Kishine, H.; Ohtsubo, Y.; Minematsu, T.; Matsubara, Y.; Okamoto, T. Studies on physiologically active substances in citrus fruit peel. Part 23. Isolation and characterization of new limonoid glycosides from Citrus unshiu peels. Carbohydr. Res. 1999, 315, 142-147. [CrossRef]

3. Baba, S.; Ueno, Y.; Kikuchi, T.; Tanaka, R.; Fujimori, K. A Limonoid Kihadanin B from Immature Citrus unshiu Peels Suppresses Adipogenesis through Repression of the Akt-FOXO1-PPAR $\gamma$ Axis in Adipocytes. J. Agric. Food Chem. 2016, 64, 9607-9615. [CrossRef] [PubMed]

4. Kim, D.K.; Lee, K.T.; Eun, J.S.; Zee, O.P.; Lim, J.P.; Eum, S.S.; Kim, S.H.; Shin, T.Y. Anti-allergic components from the peels of Citrus unshiu. Arch. Pharm. Res. 1999, 22, 642-645. [CrossRef] [PubMed]

5. Sawabe, A.; Matsubara, Y.; Iizuka, Y.; Okamoto, K. Studies on physiologically active substances in citrus fruit peel. Part XIV. Structures and hypotensive effect of flavonoid glycosides in young Citrus unshiu peelings. Yukagaku 1989, 38, 53-59.

6. Yang, X.; Kang, S.-M.; Jeon, B.-T.; Kim, Y.-D.; Ha, J.-H.; Kim, Y.-T.; Jeon, Y.-J. Isolation and identification of an antioxidant flavonoid compound from citrus-processing by-product. J. Sci. Food Agric. 2011, 91, 1925-1927. [CrossRef] [PubMed]

7. Shin, H.J.; Nam, J.-W.; Yoon, U.J.; Han, A.-R.; Seo, E.-K. Identification of three new flavonoids from the peels of Citrus unshiu. Helv. Chim. Acta 2012, 95, 240-245. [CrossRef]

8. Seo, K.-H.; Lee, D.-Y.; Nam, T.-G.; Kim, D.-O.; Lee, D.-G.; Kim, E.-K.; Kang, H.-C.; Song, M.-C.; Baek, N.-I. New tocopherol analogue with radical-scavenging activity from the peels of Citrus unshiu Marcovich. J. Korean Soc. Appl. Biol. Chem. 2013, 56, 747-750. [CrossRef]

9. Seo, K.H.; Lee, D.Y.; Lee, D.S.; Kang, H.C.; Kim, H.S.; Kim, Y.C.; Baek, N.I. Hepatoprotective and neuroprotective tocopherol analogs isolated from the peels of Citrus unshiu Marcovich. Nat. Prod. Res. 2015, 29, 571-573. [CrossRef] [PubMed]

10. Yoshikawa, K.; Kobayashi, M.; Arihara, S. Flower fragrance precursors from flower buds of Citrus unshiu MARCOV. Nat. Med. 1996, 50, 176-178.

11. Eom, H.J.; Lee, D.; Lee, S.; Noh, H.J.; Hyun, J.W.; Yi, P.H.; Kang, K.S.; Kim, K.H. Flavonoids and a Limonoid from the Fruits of Citrus unshiu and Their Biological Activity. J. Agric. Food Chem. 2016, 64, 7171-7178. [CrossRef] [PubMed]

12. Noh, H.J.; Hwang, D.; Lee, E.S.; Hyun, J.W.; Yi, P.H.; Kim, G.S.; Lee, S.E.; Pang, C.; Park, Y.J.; Chung, K.H.; et al. Anti-inflammatory activity of a new cyclic peptide, citrusin XI, isolated from the fruits of Citrus unshiu. J. Ethnopharmacol. 2015, 163, 106-112. [CrossRef] [PubMed]

13. Oh, H.J.; Ahn, H.M.; Kim, S.S.; Yun, P.Y.; Jeon, G.L.; Ko, Y.H.; Riu, K.Z. Composition and antimicrobial activities of essential oils in the peel of citrus fruits. J. Appl. Biol. Chem. 2007, 50, 148-154.

14. Nakatani, M.; Takao, H.; Iwashita, T.; Naoki, H.; Hase, T. The structure of graucin A, a new bitter limonoid from Evodia grauca Miq. (Rutaceae). Bull. Chem. Soc. Jpn. 1987, 60, 2503-2507. [CrossRef]

15. Min, Y.D.; Kwon, H.C.; Yang, M.C.; Lee, K.H.; Choi, S.U.; Lee, K.R. Isolation of limonoids and alkaloids from Phellodendron amurense and their multidrug resistance (MDR) reversal activity. Arch. Pharm. Res. 2007, 30, 58-63. [CrossRef] [PubMed]

16. Kondo, Y.; Suzuki, H.; Nozoe, S. Two $\gamma$-hydroxybutenolides from the bark of Phellodendron amurense and photooxidation of limonoids. Yakugaku Zasshi 1985, 105, 742-746. [CrossRef]

17. Lee, S.-Y.; Morita, H.; Takeya, K.; Itokawa, H.; Fukaya, H. Limonoids from Citrus nippokoreana. Nat. Med. 1999, 53, 255-258.

18. Yang, X.-W.; Zhang, H.; Hu, J. Isolation and structure characterization of evolimorutanin from the unripe fruits of Evodia rutaecarpa var. bodinieri. Fenxi Huaxue 2008, 36, 219-222.

19. Garcez, F.R.; Garcez, W.S.; Tsutsumi, M.T.; Roque, N.F. Limonoids from Trichilia elegans ssp. elegans. Phytochemistry 1997, 45, 141-148. [CrossRef] 
20. Liu, J. Two new limonoids from Polygonum orientale L. Indian J. Chem. Sect. B Org. Chem. Incl. Med. Chem. 2001, 40, 644-646.

21. Khalil, A.T.; Maatooq, G.T.; El Sayed, K.A. Limonoids from Citrus reticulata. Z. Naturforsch. C J. Biosci. 2003, 58, 165-170. [CrossRef]

22. Bennett, R.D.; Hasegawa, S. Limonoids of calamondin seeds. Tetrahedron 1981, 37, 17-24. [CrossRef]

23. Nakagawa, H.; Takaishi, Y.; Tanaka, N.; Tsuchiya, K.; Shibata, H.; Higuti, T. Chemical Constituents from the Peels of Citrus sudachi. J. Nat. Prod. 2006, 69, 1177-1179. [CrossRef] [PubMed]

24. Zhang, Y.; Liu, C.; Dong, B.; Ma, X.; Hou, L.; Cao, X.; Wang, C. Anti-inflammatory activity and mechanism of surfactin in lipopolysaccharide-activated macrophages. Inflammation 2015, 38, 756-764. [CrossRef] [PubMed]

25. Yamada, T.; Muroga, Y.; Jinno, M.; Kajimoto, T.; Usami, Y.; Numata, A.; Tanaka, R. New class azaphilone produced by a marine fish-derived Chaetomium globosum. The stereochemistry and biological activities. Bioorg. Med. Chem. 2011, 19, 4106-4113. [CrossRef] [PubMed]

26. Matsui, Y.; Kikuchi, T.; Inoue, T.; Muraoka, O.; Yamada, T.; Tanaka, R. Carapanolides J-L from the seeds of Carapa guianensis (Andiroba) and their effects on LPS-activated NO production. Molecules 2014, 19, 17130-17140. [CrossRef] [PubMed]

Sample Availability: Not available. 